\title{
Knowledge and Practice regarding Self-Care among the patients with type II Diabetes of Kapan,Kathmandu
}

\author{
Saraswati Sharma ${ }^{1}$ \& Sushila Devi Bhandari ${ }^{2}$ \\ ${ }^{1}$ Ph.D Scholar, Mewar University, Chittorgar (Rajasthan), India \\ ${ }^{2}$ Ph.D Scholar, Mewar University, Chittorgar (Rajasthan), India
}

\section{Corresponding Author}

Saraswati Sharma

Email: sharmasaraswati505@yahoo.com.

\begin{abstract}
Introduction: Diabetes is considered as one of the chronic health problems and the 6th leading cause of mortality in the world. Self-management of diabetes involves a number of considerations and choices that the patient with Diabetes must make on a daily basis. It requires that patients are able to reconcile their resources, values and preferences with a therapeutic of a healthy diet, exercise, no smoking, low or no alcohol intake, glucose monitoring and for some patients, medication. Objective: To assess the knowledge regarding Self-Care and practice among the patients with type II Diabetes in in kapan muncipality kathmandu. Methods: Descriptive cross sectional study was adopted to assess the knowledge regarding Self-Care and the practice among the patients with type II Diabetes. The study population was 100and study period was From 2014 September 15-October15 in Kapan, Kathmandu. Result: The study was carried out in 100 respondents among them the majority $30 \%$ respondent have adequate knowledge and $12 \%$ respondent have inadequate knowledge. In these respondents practice seem to be sufficient. Among the 58 respondent, majority of the respondents (38\%) have insufficient knowledge and (20\%) have sufficient. Knowledge and in these respondents practice seems to be inadequate. The result shows that majority (58\%) of the respondents has inadequate practice and (42\%) had adequate practice. There was equal percentage of sufficient and insufficient knowledge among respondents i.e. 50\%.Conclusion: The present study concluded that respondents who have sufficient knowledge have adequate practice about self-care majority of the respondents having insufficient knowledge have inadequate practice.
\end{abstract}

KEY WORDS: Diabetes Mellitus, Knowledge, Practice, Self care

\section{INTRODUCTION}

Diabetes Mellitus is a group of metabolic diseases characterized by increased levels of glucose in the blood (hyperglycemia) resulting from defects in insulin secretions, insulin action, or both (International Diabetes Federation, 2009).Current predictions estimate that the prevalence of diabetes will reach 438 million by 2030 and that $80 \%$ of prevalent cases will occur in the developing world (World Health Organization, 2012).Five and a half million deaths in 
2013 worldwide i.e.1 patient/six seconds have been occurring due to diabetes. (International Diabetes Federation, 2013).Self-Care means monitoring blood glucose levels, taking medication, maintaining a healthy diet, regular exercise and self foot exam. The estimates show that $76 \%$ of global health expenditure on diabetes in 2013 was for people between the ages of 50 and 79 years. The treatment expenses on development of complications is high thus to focus diabetic patients on self-care in order to prevent that situation. Diabetes caused at least USD548 billion in health expenditure in 2013 i.e.11\% of total health spending on adults (International Diabetes Federation, 2013).

Hence study on diabetes self-care seems prominent to reduce risk of disease and great extent of self-control and prevention. Diabetic Patient towards Self Care Activities for Longevity of Life' at M.L.B. Medical College, Jhansi among 500 diabetic patients indicated that mean score (9.44) with S.D of 2.06 and this value indicates that the practice was not satisfactory and no significant association between practice towards self-care activities with socio demographic variables such as gender, age, marital status, type of the family, educational status, dietary habits and duration of diabetes. (Singh \& Vijij, 2014). Among self-care practices, following a controlled diet $(\mathrm{p}=0.04)$, regular exercise $(\mathrm{p}=0.04)$ and compliance with drugs $(\mathrm{p}=$ 0.03) were significantly associated with the achieving glycemic control (Padma, Bele, Bodhare, \& Valsangkar, 2012).

Nearly half of diabetic patients had poor self-care practices and an educational status was associated with self-care (Amente, Belachew, Hailu, \& Berhanu, 2014)

\section{METHODS}

This is a descriptive cross sectional purposive study design. Non-probability purposive sampling technique was used to explore the knowledge and practice regarding Diabetes Mellitus. Study was performed at Shanti Tole ward no: 2 Kapan, Kathmandu. Over a period of one month that is (from September 15-October15). Age of the respondents was 40 -60 years. Clients with severe diabetes complication, type I diabetes were excluded from the study. For ethical consideration formal permission was taken from the concerned authority of study area by submitting an official letter. The objective and purpose of the study was clearly explained before data collection to the study subject. Informed consent with individual was taken before the data collection. Privacy, confidentiality and anonymity of the respondent were maintained. Semi-structured questionnaire was used in the interview schedule.

\section{Scoring for knowledge and practice (manage level)}

The scoring criteria are as below:

For knowledge,

Below mean $(<14.1$ or $<52 \%)$ - Insufficient knowledge

Above or equal to mean $(\geq 14.1$ or $\geq 52 \%)$ - Sufficient knowledge

For practice,

Below mean $(<14.4$ or $<51 \%)$ - Inadequate practice

Above or equal to mean ( $\geq 14.4$ or $\geq 51 \%$ ) - Adequate practice (Hanu, et al., 2013). 


\section{RESULTS}

The survey was conducted in 100 clients. Frequency and percentage were used and interpreted through the help of table and figures to make the data clearer and more understandable.

1. Socio-demographic characteristics of respondents

Majority of the respondents are of age group $46-50$ and 56 - 60 contributing (32\%) each. Regarding gender (56\%) is male and (88\%) are from Newar ethnic group. Majority of respondents $(52 \%)$ were from Hindu religion. Majority of the respondents (96\%) were married. Most of the respondents (78\%) are literate and (32\%) are engaged in agriculture and other (32\%) related to other occupations. Family income of respondents shows that (36\%) of respondents fall between the maximum range of 10,000 -15,000.

2. Finding related to knowledge about self-care

\subsection{Self-care knowledge related to Diabetic diet plan and intake of alcohol and smoking:}

Among 100 respondents majority of the respondents (86\%) have the knowledge necessity of blood test for Diabetes Mellitus and Diabetic diet plan. (98\%) have the knowledge that smoking and intake of alcohol should be strictly avoided in Diabetes Mellitus.

\subsection{Self-care Knowledge related to exercise and duration of exercise:}

Among 100 respondents majority of the respondent (96\%) have the knowledge concerning the need of the exercise to be done. Majority of the respondents (78\%) know that the exercise should be practiced at least 30 minutes.

3. Knowledge about regular medicine, check-up and blood test :

Majority of the respondents $(96 \%)$ have the knowledge that if medicine is prescribed by the doctor should be taken regularly, (88\%) respondents have the knowledge regular check-up and $(68 \%)$ have the knowledge about importance of the regular blood test.

4. Diabetic Self-care Knowledge related to foot care:

Majority of the respondent (94\%) of the respondents have the knowledge of the necessity of foot care. Among them (30\%) said it should be washed daily, (40\%) that it should be washed twice a day and $(24 \%)$ said that it should be washed daily also said should also be washed every time when you comeback from outside.

5. Diabetic self-care knowledge related to carrying diabetic identity card

Majority of the respondent (92\%) have heard about ID card its importance of carrying it.

6. Finding related to self-care practices done Diabetic client 
TABLE 1: Practices that are followed by the Respondents

\begin{tabular}{lll} 
& & n=100 \\
\hline Practices & Frequency & Percent \\
\hline Food intake per day & 20 & $20 \%$ \\
$\quad$ Two times & 42 & $42 \%$ \\
Three times & 38 & $38 \%$ \\
Four times & & \\
Exercise frequency & 72 & $72 \%$ \\
Daily & 18 & $18 \%$ \\
Two to three days in a week & 10 & $10 \%$ \\
Four to five days in a week & & \\
Exercise duration per time & 22 & $22 \%$ \\
20 minutes & 30 & $30 \%$ \\
30 minutes & 48 & $48 \%$ \\
60 minutes & & \\
Physical checkup frequency & 22 & $22 \%$ \\
Half yearly & 78 & $78 \%$ \\
Once in a year & & \\
Blood glucose test frequency & 2 & $2 \%$ \\
Once in a week & 82 & $16 \%$ \\
Once in a month & 16 & $100 \%$ \\
Half yearly & 100 &
\end{tabular}

Sources: It is primary data and it is collected from the respondent who are suffering from type II Diabetes from Shanti tole, Kapan from ward no: 2 through structured and semi structured questionnaire by interview schedule, with each respondent on from September 15-October, 2014. As in the above table, food intake in a day, most respondents (42\%) was 3 times per day, only (20\%) ate twice a day found to be insufficient food intake practice besides (38\%) took meal 4 times a day. Nearly $3 / 4^{\text {th }}$ of respondents exercised daily to control diabetes, (10\%) of them found to have satisfactory exercise practice which is four to five days in a week and rest $(18 \%)$ had poor practice only up to 3 days in a week. Considering duration of exercise (78\%) of respondents had efficiently practiced. Most of the diabetes patients in the study (78\%) had checked physical health only once in a year which is not sufficient. In contrast blood glucose test performance frequency of all the respondents found to be satisfactory i.e. they all checked blood glucose level within six months. 


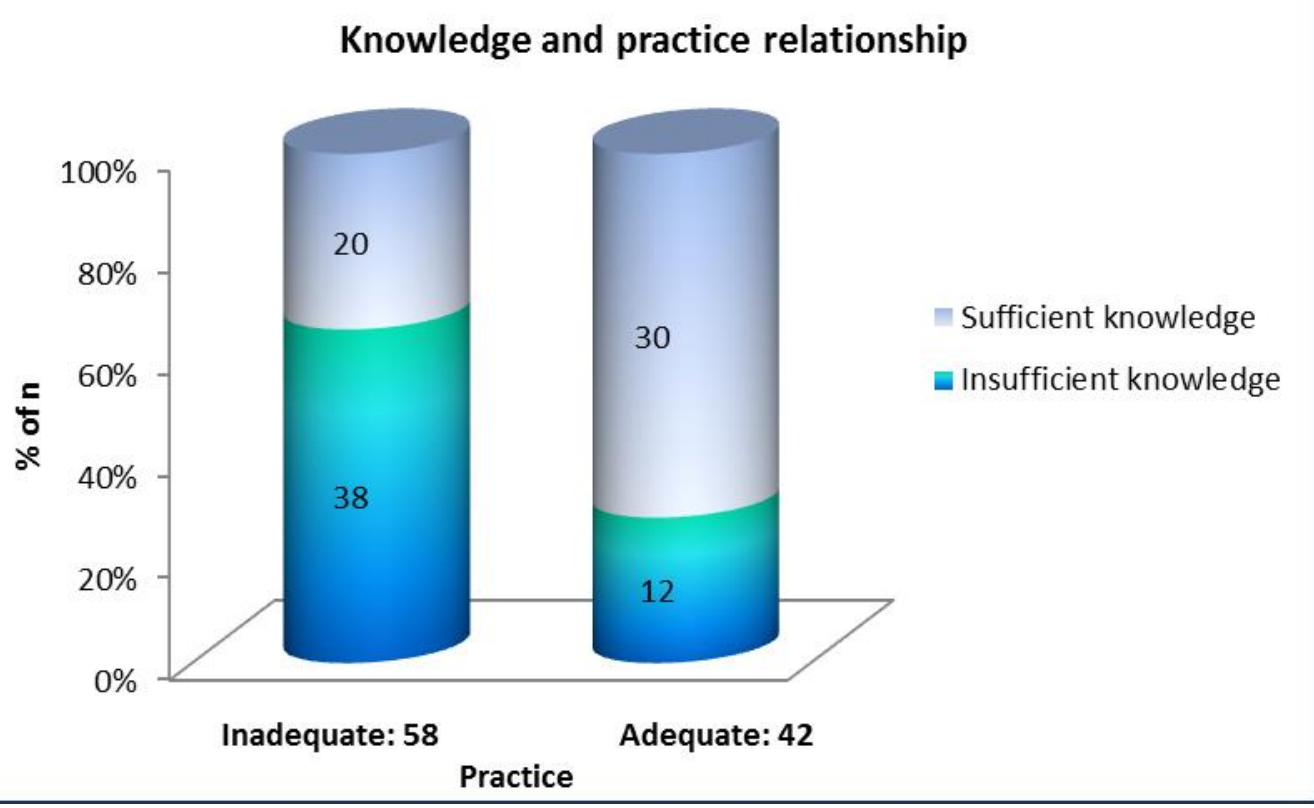

Sources: Data is collected from ward no: 2 Shantitole, kapan by the help of structured and semi structured questionnaire with interview schedule. The respondents were Diabetes client. Datas were collected with each respondent by purposive non probability sampling technique from september15-October, 2014.

As illustrated in the above figure, the result displayed pointing to majority that the respondents having insufficient knowledge have inadequate practices those were $38(76 \%)$ out of 58 respondents. In addition patients having adequate knowledge tended to practice efficiently i.e. $30(60 \%)$ out of 42 respondents practicing adequately. In contrary, 20 (40\%) sufficient knowledgeable respondents seemed to have inadequate practice and 12 (24\%) insufficient knowledge having respondents seemed to practice well. The p-value for this test is 0.010 that indicated positive relationship between knowledge and practice when tested in $5 \%$ significance level.

\section{DISCUSSIONS}

Knowledge and practice is based on the mean score of total respondents and divided into two halves. The mean of knowledge was 28.2 and 28.8 for practice. This method is consistent with the method used by with "Knowledge and Practices. Among People with Diabetes Attending a Secondary Care Rural Hospital in Southern India" by Hanu et al., the total and mean knowledge scores and practices scores were calculated. The study participants were categorized into two groups: Those who scored mean or above as having good knowledge and good practices and those with less than mean scores as having poor knowledge and poor practices.

Among 100 respondents, having insufficient knowledge, $76 \%$ practiced inadequately. Similar study conducted by Pandey \& Chapagain, in 2013 in Metro polyclinic Kathmandu, Nepal also stated that out of 50 respondents unsatisfactory self-care practice was more than half 
$(56.75 \%)$ among adequate knowledge respondents. Among respondents having inadequate knowledge, majority $(92.31 \%)$ had unsatisfactory practice. Majority (74\%) had adequate knowledge and satisfactory self-care practice was done by only study $34 \%$.The study result is consistent with the present study.

The quantified result revealed that $50(50 \%)$ out of total 100 respondents were found to have adequate self-care knowledge and 50(50\%) have inadequate knowledge. According to similar study, done by Jackson, Adibe, Okonta, \& Ukwe, in 2014, 79.5\% had 70\% knowledge about self-care. The study result does not consistent with the present study it may be due to study done in different country.

Majority of the respondent (94\%) of the respondents have the knowledge of the necessity of foot care. Among them $30 \%$ said it should be washed daily, $40 \%$ said that it should be washed twice a day and $24 \%$ said that it should be washed daily and also said should also wash every time when comeback from outside. Similar type of study done by (Saleh, Mumu, Ara, Begum, \& Ali, 2012)81\% with good knowledge and about $70 \%$ from average and poor groups did not take care of their feet. The result is consistent with the present study.

\section{CONCLUSION}

It can be concluded that out of the total 100 respondents, the mean score for knowledge was 14.1 i.e. $52 \%$ of maximum score 27 . The total number of sufficient and insufficient knowledge is exactly half .Similarly, the mean score for practice was 14.4 i.e. $51 \%$ of maximum score of 28. Among them with inadequate and adequate practice were $58 \%$ and $42 \%$ respectively. Statistically significant associations were established between the level of knowledge and the practice $(\mathrm{p}<0.001)$.If we give more knowledge about self-care to diabetes patient encourage them to practice it, the national budget that is being spent for treating Diabetes related complication will be reduced and this budget can be spent for other health sector and to some extent we can upgrade the health condition of our country people.

\section{ACKNOWLEDGEMENT}

I would like to extend my gratitude to my supervisor professor Dr. Sarala Joshi for her continuous support and guidance. I would like to express my sincere thanks to $\mathrm{PhD}$ Centre for providing greater forum to publish this article. My special thanks go to Staffs of PhD Centre for their support and guidance. I am equally thankful to all the participants of my study. At last but not the least I would like to thank the respondents of this study in which without their help, cooperation and participation this study would not have been possible.

\section{BIBLOGRAPHY}

Amente, T., Belachew, T., Hailu, E., \& Berhanu, N. (2014). World Journal of Medicine and Medical Science. Self Care Practice And Its Predictors Among Adults With Diabetes Mellitus. 
Hanu, G., PS, R., Manjunath, K., Reginal, A., Abraham, V. J., Kuryan, G., et al. (2013, March). Foot Care Knowledge and Practices and the Prevalence of Peripheral Neuropathy Among People with Diabetes Attending a Secondary Care Rural Hospital in Southern India . Journal of Family Medicine and Primary Care, 27-32.

Hasnain, S., \& Sheikh, N. H. (2009, October). Knowledge and practices regarding foot care in diabetic patients visiting diabetic clinic in Jinnah Hospital, Lahore. J Pak Med Assoc, 59(10), 687-690.

International Diabetes Federation. (2009, March 14). IDF Diabetes Atlas 2009 (4th ed.). (N. Unwin, D. Whiting, D. Gan, O. Jacqmain, \& G. Ghyoot, Eds.) IDF.

International Diabetes Federation. (2011, June). IDF. Retrieved May 5, 2014, from www.idf.org: http://www.idf.org/types-daibetes

Jackson, I. L., Adibe, O. M., Okonta, J. M., \& Ukwe, V. C. (2014, Jul). Knowledge of self-care among type 2 diabetes patients in two states of Nigeria. Pharmacy Practice, 12(3), 404.

Maskey, R., Shakya, D. R., Sharma, S. K., Karki, P., \& Lavaju, P. (2011). Diabetes mellitus related complications in out- patient clinic of tertiary care hospital. Journal of College of Medical Sciences-Nepal, 7(2), 9-16.

Nepal Diabetes Association. (2013). www.nepaldiabetesassociation.com. Retrieved May 6, 2014, from NDA website: http://www.nepaldiabetesassociation.com/research.php

Padma, K., Bele, S. D., Bodhare, T. N., \& Valsangkar, S. (2012, Janaury-March). Evaluation Of Knowledge And Self Care Practices In Diabetic Patients And Their Role In Disease Management. National Journal of Community Medicine, 3(1), 3.

Rensburg, J. v. (2009). Preventative foot care in people with diabetes: Quality patient education. JEMDSA, 14(2), 1-2.

Saleh, F., Mumu, S. J., Ara, F., Begum, H. A., \& Ali, L. (2012). Knowledge and self-care practices regarding diabetes among newly diagnosed type 2 diabetics in Bangladesh: a cross-sectional study. BMC Public Health Journal, 12(4), 11-17.

Singh, M., \& Vijij, P. T. (2014). A Study to Assess the Practice of Diabetic Patient towards Self Care Activities for Longevity of Life. Scholars Journal of Applied Medical Sciences (SJAMS), 2(1A), 57-60.

Singh, N. K., Shan, N. K., Bhandari, A., Pandey, S., \& Sharma, S. K. (2013). Presentations and complications of diabetes patients presenting to diabetic clinic of Eastern Nepal. Journal of College of Medical Sciences-Nepal, 9(3), 25-30.

World Health Organization. (2012, August). WHO. Retrieved March 10, 2014, from WHO website: www.who.int 